\title{
Early Enhanced Leucine-Rich $\alpha$-2-Glycoprotein-1 Expression in Glomerular Endothelial Cells of Type 2 Diabetic Nephropathy Model Mice
}

\author{
Sona haku, ${ }^{1}$ Hiromichi Wakui $\mathbb{D}^{1},{ }^{1}$ Kengo Azushima $\mathbb{D}^{1},{ }^{1,2}$ Kotaro Haruhara, ${ }^{1,3}$ \\ Sho Kinguchi, ${ }^{1}$ Kohji Ohki, ${ }^{1}$ Kazushi Uneda, ${ }^{1}$ Ryu Kobayashi ${ }^{1},{ }^{1}$ Miyuki Matsuda, \\ Takahiro Yamaji, ${ }^{1}$ Takayuki Yamada, ${ }^{1}$ Shintaro Minegishi, ${ }^{1}$ Tomoaki Ishigami, ${ }^{1}$ \\ Akio Yamashita, ${ }^{4}$ Kenichi Ohashi, ${ }^{5}$ and Kouichi Tamura ${ }^{10}{ }^{1}$ \\ ${ }^{1}$ Department of Medical Science and Cardiorenal Medicine, Yokohama City University Graduate School of Medicine, Yokohama, Japan \\ ${ }^{2}$ Cardiovascular and Metabolic Disorders Program, Duke-NUS Medical School, Singapore \\ ${ }^{3}$ Division of Nephrology and Hypertension, Department of Internal Medicine, The Jikei University School of Medicine, Tokyo, Japan \\ ${ }^{4}$ Department of Molecular Biology, Yokohama City University Graduate School of Medicine, Yokohama, Japan \\ ${ }^{5}$ Department of Molecular Pathology, Yokohama City University Graduate School of Medicine, Yokohama, Japan
}

Correspondence should be addressed to Hiromichi Wakui; hiro1234@yokohama-cu.ac.jp and

Kengo Azushima; azushima@yokohama-cu.ac.jp

Received 16 August 2018; Accepted 3 October 2018; Published 1 November 2018

Guest Editor: Edyta Sutkowska

Copyright ( 2018 Sona haku et al. This is an open access article distributed under the Creative Commons Attribution License, which permits unrestricted use, distribution, and reproduction in any medium, provided the original work is properly cited.

\begin{abstract}
Abnormal angiogenesis plays a major role in the development of early stage diabetic nephropathy. Vascular endothelial growth factor (VEGF) is a classical proangiogenic factor that regulates abnormal glomerular angiogenesis linked to glomerular hypertrophy in the early stage of diabetic nephropathy. Leucine-rich $\alpha$-2-glycoprotein-1 (LRG1) was recently reported as a novel proangiogenic factor that is expressed in endothelial cells and promotes angiogenesis by modulating the transforming growth factor- $\beta$ signaling pathway. However, the pathophysiology of LRG1 in diabetic nephropathy remains largely unknown. In the present study, we investigated intrarenal expression of the novel proangiogenic factor LRG1 in diabetic $d b / d b$ mice by immunohistochemistry and a laser capture microdissection method during the development of diabetic nephropathy. We hypothesized that glomerular LRG1 expression is increased earlier than VEGF expression under conditions of pathological angiogenesis in the early stage of diabetic nephropathy. Thus, we compared glomerular expression of VEGF and LRG1 in diabetic $d b / d b$ mice at 16 and 24 weeks of age. At 16 weeks, diabetic $d b / d b$ mice exhibited glomerular hypertrophy with abnormal angiogenesis characterized by endothelial cell proliferation, which was concomitant with an increase in LRG1 expression of glomerular endothelial cells. However, glomerular VEGF expression was not increased at this early stage. At 24 weeks, the features of early diabetic nephropathy in $d b / d b$ mice had developed further, along with further enhanced glomerular LRG1 expression. At this late stage, glomerular VEGF and fibrosisrelated-gene expression was also significantly increased compared with nondiabetic $d b / m$ mice. These results suggest that LRG1 plays a pivotal role in the initial development of diabetic nephropathy by promoting abnormal angiogenesis, thereby suggesting that LRG1 is a potential preemptive therapeutic target of diabetic nephropathy.
\end{abstract}

\section{Introduction}

Diabetic nephropathy has become a leading cause of end stage renal disease (ESRD) worldwide $[1,2]$. Despite treatment by inhibition of the renin angiotensin system and tight glycemic control, the risk of ESRD remains high.
Diabetic nephropathy is diagnosed at the early stage by detection of microalbuminuria. However, early prevention of diabetic nephropathy progression remains challenging. Therefore, understanding the pathogenesis of early stage diabetic nephropathy and developing methods to control its progression are important issues. 
Abnormal angiogenesis plays a major role in the development of early stage diabetic nephropathy $[3,4]$. It is associated with glomerular hypertrophy and urinary albumin excretion [5-7]. Morphological changes in capillaries, such as elongation and an increased number, contribute to glomerular hypertrophy $[3,4]$. In these aberrant vessels, the endothelial cells are swollen and immature, leading to increased vascular permeability $[5,8,9]$.

Vascular endothelial growth factor (VEGF) is a critical regulator of abnormal angiogenesis, and its glomerular expression is involved in the pathogenesis of early stage diabetic nephropathy [10-12]. VEGF is mainly expressed in podocytes, and its receptor, vascular endothelial growth factor receptor-2 (VEGFR-2), is expressed in glomerular endothelial cells [13]. VEGF-VEGFR-2 signaling is upregulated in the diabetic glomerulus, which leads to abnormal angiogenesis and endothelial cell proliferation [14].

Very recently, Leucine-rich $\alpha$-2-glycoprotein-1 (LRG1), a novel proangiogenic factor expressed in endothelial cells [15], has been reported to be involved in the development of diabetic nephropathy. Transcriptomic and in vitro analyses revealed that LRG1 in glomerular endothelial cells is one of the key regulators in the development of abnormal angiogenesis at the early stage of diabetic nephropathy [16]. However, the pathophysiology of LRG1 in diabetic nephropathy is still largely unknown.

The aim of this study was to compare glomerular expression of the classical proangiogenic factor VEGF and novel proangiogenic factor LRG1 in the early stage of diabetic nephropathy. We investigated glomerular expression of VEGF and LRG1 in a mouse model of diabetes (C57BL/KsJ$\mathrm{db} / \mathrm{dbJcl} ; d b / d b$ mouse) and compared the changes in expression at 16 and 24 weeks of age to evaluate their association with diabetic nephropathy development.

\section{Materials and Methods}

2.1. Animals. This study was performed in accordance with the National Institutes of Health guidelines for the use of experimental animals. All animal experiments were reviewed and approved by the Animal Studies Committee of Yokohama City University. Male $d b / d b$ mice and their age-matched nondiabetic $d b / m$ (C57BL/KsJ) littermates were purchased from CLEA Japan. The mice were housed in a controlled environment with a 12 -hour light-dark cycle at $25^{\circ} \mathrm{C}$. The mice were allowed free access to food and water. Mice were fed a standard diet $(0.3 \% \mathrm{NaCl}, 3.6 \mathrm{kcal} / \mathrm{g}$, and $13.3 \%$ energy as fat; Oriental MF, Oriental Yeast Co, Ltd.). The mice were sacrificed at 16 and 24 weeks of age.

2.2. Biochemical Assays. Blood glucose was measured in blood obtained by tail vein puncture. Blood samples were also collected by cardiac puncture when mice were sacrificed in a fed state as described previously $[17,18]$. Whole blood was centrifuged at $800 \times g$ for 10 minutes at $4^{\circ} \mathrm{C}$ to separate the plasma. The resulting plasma was stored at $-80^{\circ} \mathrm{C}$ until use. Plasma creatinine and urinary creatinine were measured using an autoanalyzer (Hitachi 7180; Hitachi, Tokyo, Japan).
Urinary albumin was measured by an ELISA kit (Fujifilm Wako Shibayagi, Gunma, Japan).

2.3. Metabolic Cage Analysis. To collect urine, metabolic cage analysis was performed as described previously $[19,20]$. Mice were provided with free access to tap water and fed a standard diet.

2.4. Histological and Immunohistochemical Analyses. Kidneys were fixed with $4 \%$ paraformaldehyde and embedded in paraffin. Sections of $4 \mu \mathrm{m}$ in thickness were stained with periodic acid Schiff (PAS). Immunohistochemistry was performed as described previously $[17,18,21]$. Briefly, the paraffin-embedded sections were dewaxed and rehydrated. Antigen retrieval was performed by microwave heating. The sections were treated for 60 minutes with $10 \%$ normal goat serum in phosphate-buffered saline and blocked for endogenous biotin activity using an AVIDIN/BIOTIN Blocking kit (Vector Laboratories, CA, USA). The sections were then incubated with one of the following antibodies: (1) anti-LRG1 antibody (TAKARA, Japan) diluted at 1:100 or (2) anti-CD34 antibody (BD Pharmingen, Japan) diluted at 1:100. The sections were incubated for 60 minutes with biotinylated goat anti-rabbit IgG (Nichirei Corporation, Tokyo, Japan), blocked for endogenous peroxidase activity by incubation with $0.3 \% \mathrm{H}_{2} \mathrm{O}_{2}$ for 20 minutes, treated for 30 minutes with streptavidin and biotinylated peroxidase (DAKO, Heidelberg, Germany), and then exposed to hematoxylin, dehydrated, and mounted. To evaluate the glomerular area, 25 glomeruli per mice were measured and averaged. All images were acquired using a BZ-9000 microscope (Keyence).

2.5. Real-Time Quantitative PCR Analysis. Total RNA was extracted from the kidney with ISOGEN (Nippon Gene, Tokyo, Japan), and cDNA was synthesized using the SuperScript III First-Strand System (Invitrogen, Carlsbad, CA, USA). Real-time quantitative PCR (RT-qPCR) was performed by incubating the reverse transcription product with TaqMan Universal PCR Master Mix and TaqMan probes (Applied Biosystems, Foster City, CA, USA), as described previously $[22,23]$. mRNA levels were normalized to $18 \mathrm{~S}$ rRNA as a control.

2.6. Laser Capture Microdissection and Subsequent RTqPCR Analysis. Laser capture microdissection (LMD) was performed using a Leica LMD System (LMD 6000), as described previously $[24,25]$. Briefly, formalin-fixed, paraffin-embedded tissues were cut into $10 \mu \mathrm{m}$-thick sections, mounted on polyethylene terephthalate membrane slides, and stained with hematoxylin-eosin. Next, renal glomeruli were microdissected using the LMD 6000 laser microdissection microscope. In total, 350 glomeruli were microdissected from the renal cortex per mouse. Total RNA was extracted from microdissected tissue using the RNeasy FFPE Kit (Qiagen, Hilden, Germany). cDNA was synthesized using the SuperScript III First-Strand System and applied to TaqMan RT-qPCR analysis. 
TABLE 1: Body weight, blood glucose, kidney weight, plasma creatinine, and albuminuria of $d b / d b$ and $d b / m m i c e$.

\begin{tabular}{|c|c|c|c|c|}
\hline \multirow{3}{*}{ Variable } & \multicolumn{2}{|c|}{$16 \mathrm{~W}$} & \multicolumn{2}{|c|}{$24 \mathrm{~W}$} \\
\hline & $d b / m$ & $d b / d b$ & $d b / m$ & $d b / d b$ \\
\hline & $(n=6)$ & $(n=6)$ & $(\mathrm{n}=4)$ & $(n=4)$ \\
\hline Body weight (g) & $29.2 \pm 1.3$ & $48.2 \pm 0.7 * *$ & $37.0 \pm 0.5$ & $52.2 \pm 4.8 *$ \\
\hline Blood glucose (mg/dl) & $134.8 \pm 5.8$ & $>600 * *$ & $130 \pm 6.7$ & $574 \pm 26 * *$ \\
\hline Kidney weight (mg) & $182.3 \pm 6.7$ & $217.3 \pm 8.5 * *$ & $225.0 \pm 6.7$ & $263.3 \pm 5.8 * *$ \\
\hline Plasma creatinine $(\mathrm{mg} / \mathrm{dl})$ & $0.12 \pm 0.22$ & $0.11 \pm 0.1$ & $0.08 \pm 0.01$ & $0.09 \pm 0.02$ \\
\hline Albuminuria (mg/mg.Cr) & $0.25 \pm 0.02$ & $0.67 \pm 0.16 * *$ & $0.04 \pm 0.01$ & $1.05 \pm 0.53 *$ \\
\hline
\end{tabular}

All values are means \pm SEM.

$* P<0.05, * * P<0.01$, vs $d b / m$ mice at the same age, unpaired $t$-test.

2.7. Statistical Analysis. Statistical analysis was performed using GraphPad Prism software (GraphPad Software, La Jolla, CA, USA). All quantitative data are expressed as the mean \pm SEM. Differences were analyzed using the unpaired Student's $t$-test. Values of $P<0.05$ were considered as statistically significant.

\section{Results}

3.1. Characteristics of 16- and 24-Week-Old db/db Mice. Body weight, blood glucose, kidney weight, and the albuminuria level were significantly higher in $d b / d b$ mice compared with $\mathrm{db} / \mathrm{m}$ mice at 16 weeks of age (Table 1 ). At 24 weeks of age, body weight, blood glucose, kidney weight, and the albuminuria level were significantly increased in $d b / d b$ mice compared with $\mathrm{db} / \mathrm{m}$ mice. However, serum creatinine levels were identical in $d b / d b$ and $d b / m$ mice at both 16 and 24 weeks of age (Table 1).

3.2. Glomerular Hypertrophy with Endothelial Cell Proliferation in $d b / d b$ Mice. At 16 weeks of age, $d b / d b$ mice exhibited a significantly larger glomerular area compared with $\mathrm{db} / \mathrm{m}$ mice $\left(3974.0 \pm 117.6 \mu \mathrm{m}^{2}\right.$ versus $\left.5359.0 \pm 202.3 \mu \mathrm{m}^{2}, P<0.001\right)$ (Figure 1(a)). At 24 weeks of age, the glomerular area had increased further in $d b / d b$ mice compared with $d b / m$ mice $\left(3542.2 \pm 95.2 \mu \mathrm{m}^{2}\right.$ versus $\left.5689.2 \pm 182.5 \mu \mathrm{m}^{2}, P<0.0001\right)$ (Figure 1(b)). We next examined CD34 immunostaining, as an endothelial cell marker, in the glomeruli of $\mathrm{db} / \mathrm{db}$ and $\mathrm{db} / \mathrm{m}$ mice. The immunostaining analysis showed enhancement of glomerular CD34 expression in $d b / d b$ mice compared with $\mathrm{db} / \mathrm{m}$ mice at 16 weeks of age (Figure $1(\mathrm{c})$ ), and this trend became robust at 24 weeks of age (Figure $1(d))$. These increases in glomerular endothelial cells indicated that abnormal angiogenesis had occurred in the kidneys of diabetic $d b / d b$ mice $[3,26]$. To confirm the increase of glomerular CD34 expression in $d b / d b$ mice, we next examined CD34 mRNA expression in glomeruli of $d b / d b$ and $d b / \mathrm{m}$ mice using an LMD method. Glomerular CD34 mRNA expression was significantly increased in $d b / d b$ mice compared with $d b / m$ mice at 16 and 24 weeks of age (Figures 1(e) and 1(f)). These results indicate that diabetic $d b / d b$ mice exhibit glomerular hypertrophy with endothelial cell proliferation from the early stage of their lives, and these features are exacerbated along with aging.
3.3. Enhanced LRG1 Immunostaining in Glomerular Endothelial Cells of $\mathrm{db} / \mathrm{db}$ Mice at 16 Weeks of Age. We next examined LRG1 expression and distribution in the renal cortex of $d b / d b$ and $\mathrm{db} / \mathrm{m}$ mice at 16 weeks of age by immunohistochemical analysis. The results showed that $d b / d b$ mice exhibited an increase in LRG1 expression of glomerular endothelial cells, whereas LRG1 was weakly expressed in glomerular endothelial cells of $d b / m$ mice (Figures 2(a) and 2(b)). LRG1 was also weakly expressed in tubular epithelial cells of renal tubules in $d b / d b$ and $d b / m$ mice. However, LRG1 expression was not found in podocytes of $d b / d b$ and $d b / m$ mice. These results indicate that an increase in LRG1 expression of glomerular endothelial cells might be associated with the development of diabetic nephropathy in $d b / d b$ mice.

3.4. Enhanced Glomerular LRG1 mRNA Expression in $d b / d b$ Mice at 16 Weeks of Age. We next examined gene expression in glomeruli of $d b / d b$ and $d b / m$ mice at 16 weeks of age using the LMD method. Glomerular LRG1 mRNA expression was significantly increased by approximately 2.5 -fold in $d b / d b$ mice compared with $d b / m$ mice $(1.0 \pm 0.24$ versus $2.4 \pm 0.57, P<0.05$ ) (Figure 3(a)). Intriguingly, glomerular mRNA expression levels of VEGF and its main receptor, VEGFR-2, which are key mediators of abnormal angiogenesis and glomerular hypertrophy, were not increased in $d b / d b$ mice compared with $d b / m$ mice (Figures $3(\mathrm{~b})$ and $3(\mathrm{c})$ ). Furthermore, glomerular mRNA expression levels of fibrosisrelated genes, such as transforming growth factor- $\beta$ (TGF- $\beta$ ), Collagen type IV (Collagen IV), and plasminogen activator inhibitor-1 (PAI-1), were identical in $d b / d b$ and $d b / m$ mice (Figures 3(d)-3(f)). Since LRG1 directly binds to the TGF$\beta$ accessory receptor endoglin (ENG), and then the complex binds to TGF- $\beta$ receptor-II (T $\beta$ RII), we also examined glomerular mRNA expression of ENG and T $\beta$ RII in $d b / d b$ and $\mathrm{db} / \mathrm{m}$ mice. However, there was no significant difference in glomerular mRNA expression levels of ENG and T $\beta$ RII between $d b / d b$ and $d b / m$ mice (Figures $3(\mathrm{~g})$ and $3(\mathrm{~h})$ ). These results showed that the increase in LRG1 expression preceded the increase in expression of other angiogenesis- and fibrosisrelated genes in the glomerulus of diabetic $d b / d b$ mice.

3.5. Enhanced LRG1 Immunostaining in Glomerular Endothelial Cells of $\mathrm{db} / \mathrm{db}$ Mice at 24 Weeks of Age. We next examined LRG1 expression in the renal cortex of $d b / d b$ and $d b / m$ mice 


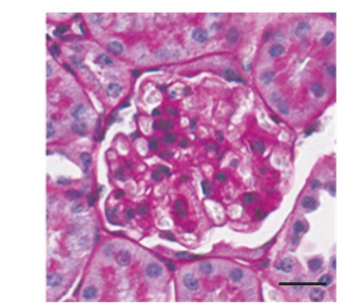

$\mathrm{db} / \mathrm{m}$

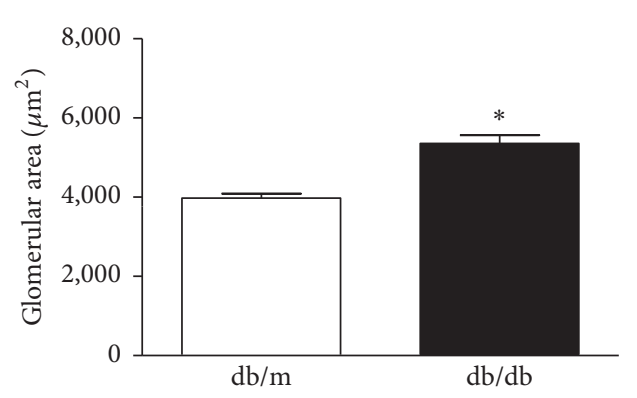

(a)

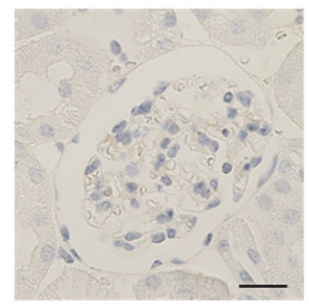

$\mathrm{db} / \mathrm{m}$

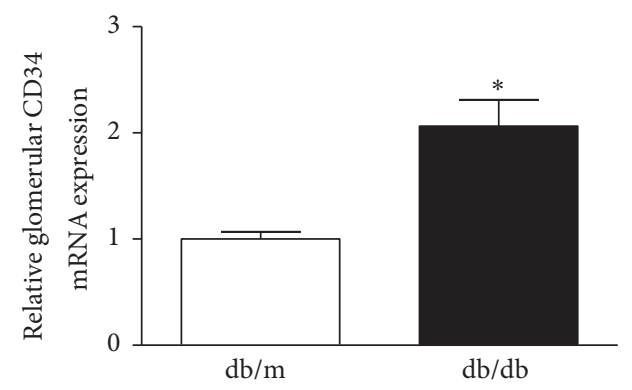

(e)

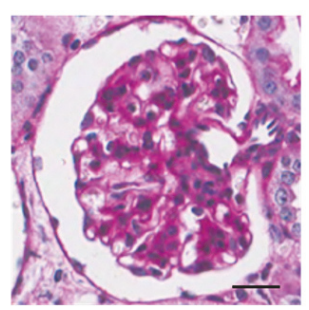

$\mathrm{db} / \mathrm{db}$

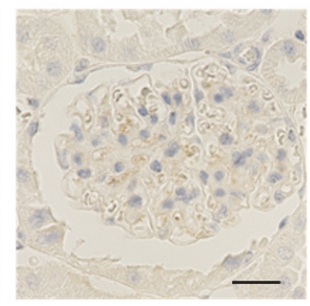

$\mathrm{db} / \mathrm{db}$

(c)

)

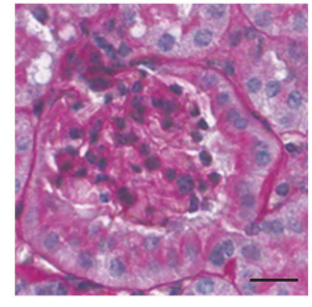

$\mathrm{db} / \mathrm{m}$

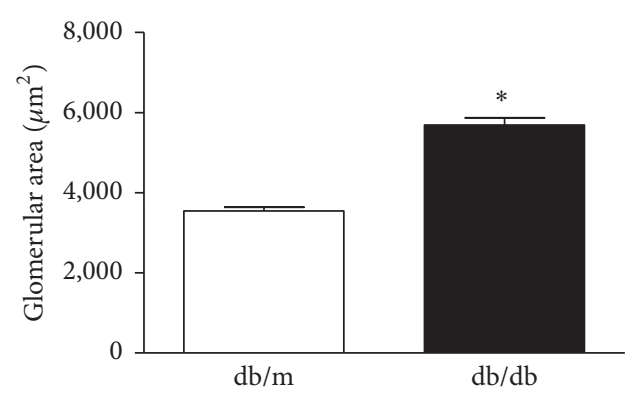

(b)

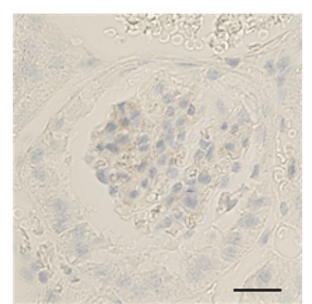

$\mathrm{db} / \mathrm{m}$

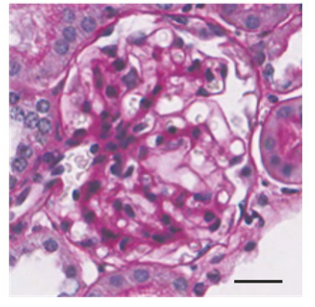

$\mathrm{db} / \mathrm{db}$

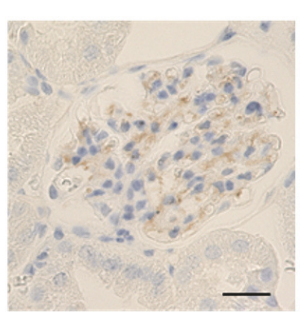

$\mathrm{db} / \mathrm{db}$

(d)

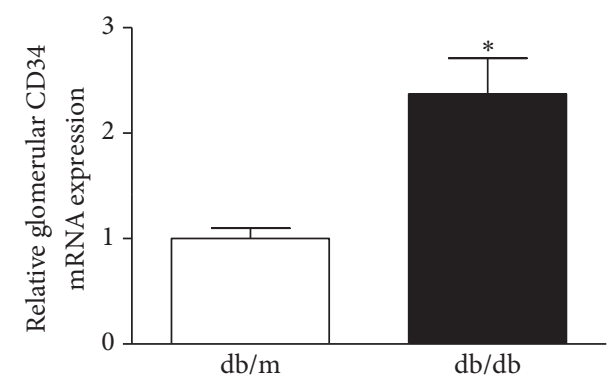

(f)

FIGURE 1: Glomerular hypertrophy with endothelial cell proliferation in $\boldsymbol{d} \boldsymbol{b} / \boldsymbol{d} \boldsymbol{b}$ mice. Representative image of PAS staining in glomeruli of $d b / m$ and $d b / d b$ mice at (a) 16 weeks and (b) 24 weeks of age. The glomerular area was increased in $d b / d b$ mice at both ages (25 glomeruli per mice were measured and averaged). Values are expressed as the mean \pm SEM ( $\mathrm{n}=4-6$ in each group). $* P<0.05$ versus $d b / m$, unpaired $t$-test. Original magnification: $\times 200$, bars $=20 \mu \mathrm{m}$. Representative image of CD34 immunostaining at (c) 16 weeks and (d) 24 weeks of age. Quantitative analysis of CD34 in 350 glomeruli identified by an LMD method at (e) 16 weeks and (f) 24 weeks of age. Values are expressed as the mean \pm SEM ( $\mathrm{n}=4-6$ in each group). $* P<0.05$ versus $d b / m$, unpaired $t$-test.

at 24 weeks of age by immunohistochemical analysis. The results showed that LRG1 expression was further enhanced in glomerular endothelial cells of $d b / d b$ mice compared with $d b / m$ mice at 24 weeks of age (Figures $4(a)$ and $4(\mathrm{~b})$ ).

3.6. Increases in Glomerular LRG1, VEGF, and Fibrosis-Related Gene Expression of $d b / d b$ Mice at 24 Weeks of Age. Finally, we examined gene expression in glomeruli of $d b / d b$ and $d b / m$ mice at 24 weeks of age. Glomerular LRG1 mRNA expression was further increased by approximately 3.5 -fold in $d b / d b$ mice compared with $\mathrm{db} / \mathrm{m}$ mice $(1.0 \pm 0.21$ versus $3.40 \pm 0.48$, $P<0.01$ ) (Figure 5(a)). Glomerular VEGF and VEGFR-2 mRNA expression was also significantly increased in $d b / d b$ mice compared with $d b / m$ mice (Figures $5(b)$ and $5(c)$ ). In 


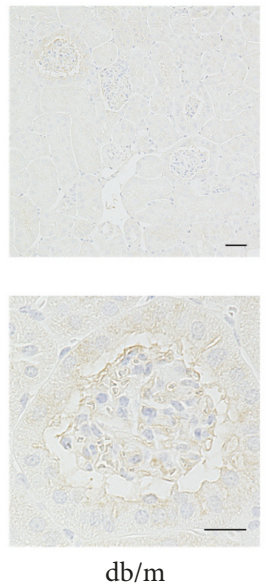

(a)
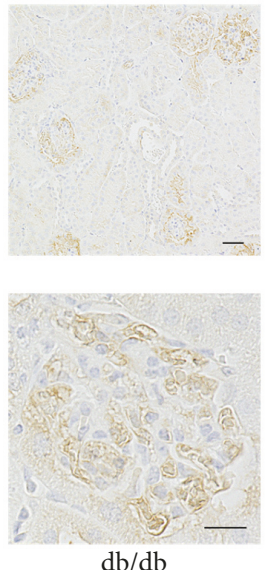

(b)

FIGURE 2: Enhanced LRG1 immunostaining in glomerular endothelial cells of $\boldsymbol{d} \boldsymbol{b} / \boldsymbol{d} \boldsymbol{b}$ mice at 16 weeks of age. Representative image of LRG1 immunostaining at 16 weeks of age in (a) $d b / m$ and (b) $d b / d b$ mice. Lower panels are higher magnifications of immunohistochemical staining for LRG1 in glomerular endothelial cells. Upper panels: original magnification: $\times 40$, bars $=20 \mu \mathrm{m}$. Lower panels: original magnification: $\times 400$, bars $=40 \mu \mathrm{m}$.

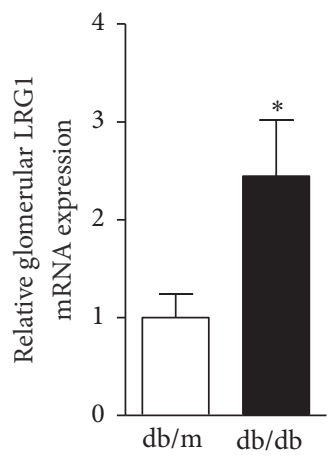

(a)

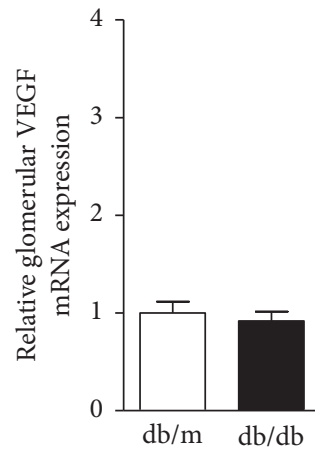

(b)

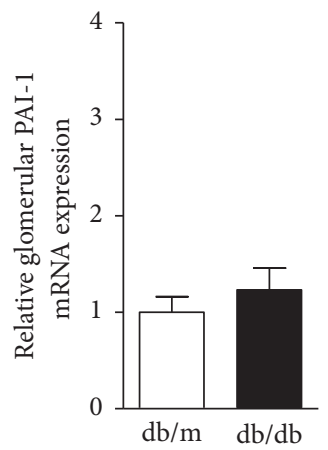

(f)

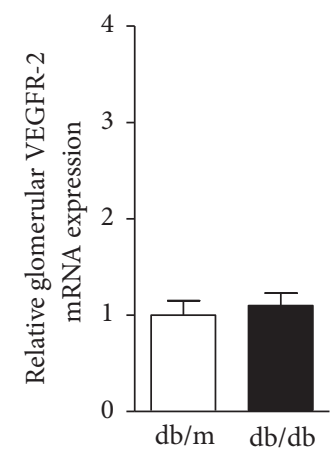

(c)

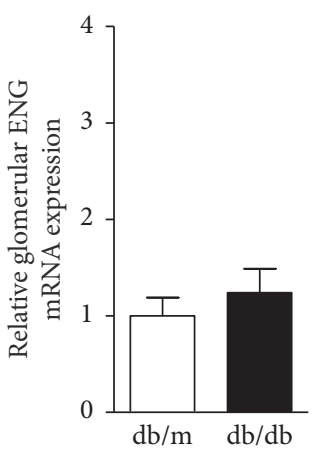

(g)

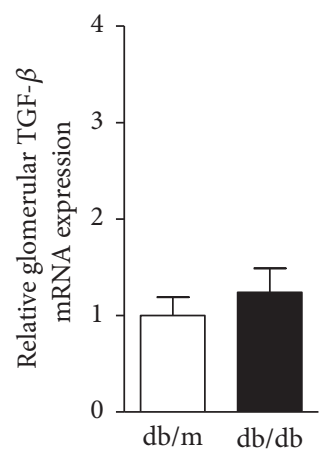

(d)

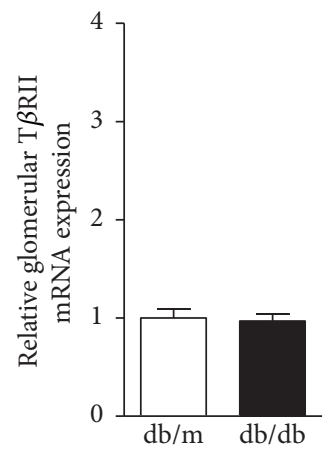

(h)

FIGURE 3: Enhanced glomerular LRG1 mRNA expression in $\boldsymbol{d} \boldsymbol{b} / \boldsymbol{d} \boldsymbol{b}$ mice at 16 weeks of age. Quantitative analysis of angiogenesis- and fibrosis-related gene expression in glomeruli identified by the LMD method in $d b / m$ and $d b / d b$ mice at 16 weeks of age. (a) LRG1, (b) VEGF, (c) VEGFR-2, (d) TFG- $\beta$, (e) Collagen IV, (f) PAI-1, (g) ENG, and (h) T $\beta$ RII. Values are expressed as the mean \pm SEM (n $=4-6$ in each group). $* P<0.05$, versus $d b / m$ mice, unpaired $t$-test. 


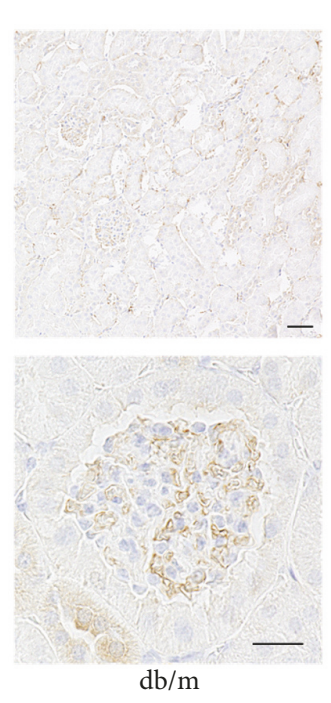

(a)

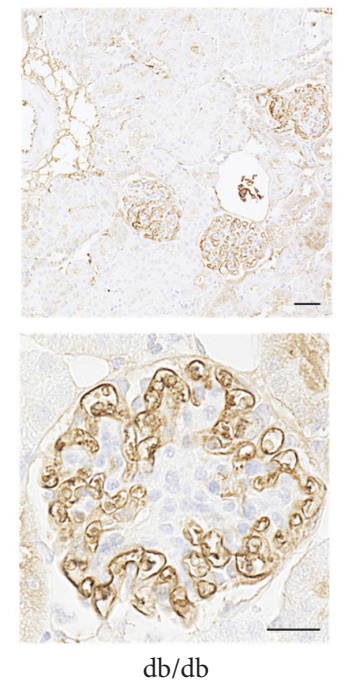

(b)

FIGURE 4: Enhanced LRG1 immunostaining in glomerular endothelial cells of $\boldsymbol{d} \boldsymbol{b} / \boldsymbol{d} \boldsymbol{b}$ mice at 24 weeks of age. Representative image of LRG1 immunostaining at 24 weeks of age in (a) $d b / m$ and (b) $d b / d b$ mice. Lower panels represent higher magnifications of immunohistochemical staining for LRG1 in glomerular endothelial cells. Upper panels: original magnification: $\times 40$, bars $=20 \mu$ m. Lower panels: original magnification: $\times 400$, bars $=40 \mu \mathrm{m}$.

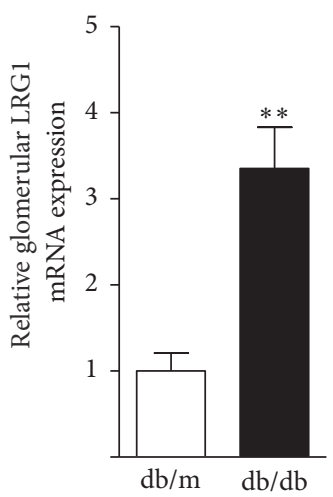

(a)

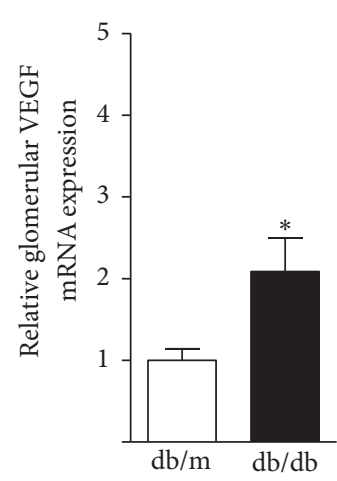

(b)

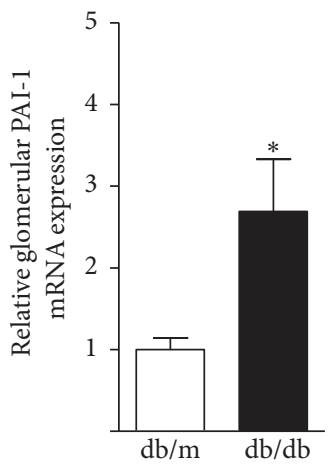

(f)

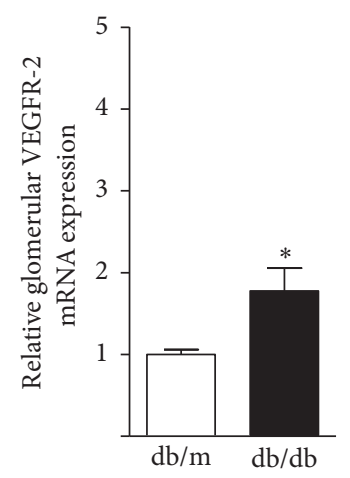

(c)

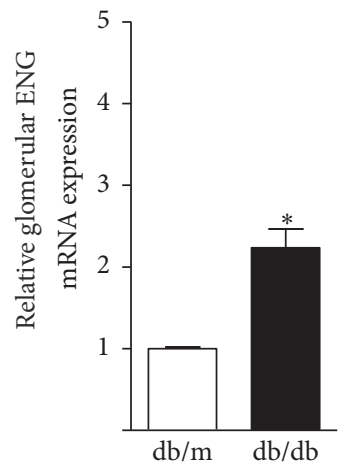

(g)

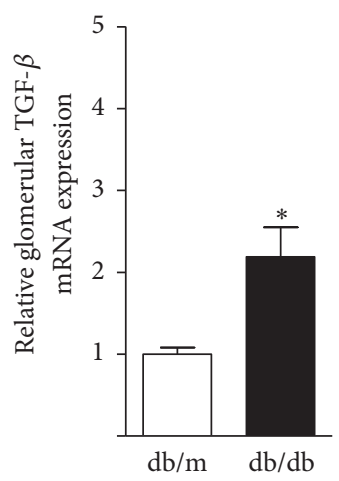

(d)

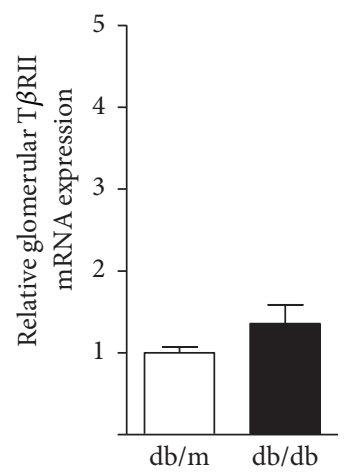

(h)

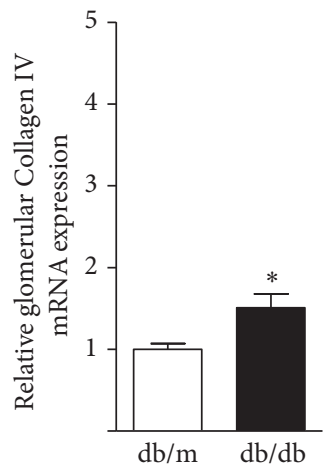

(e)

FIGURE 5: Enhanced glomerular LRG1, VEGF, and fibrosis-related gene expression in $\boldsymbol{d} b / \boldsymbol{d} \boldsymbol{b}$ mice at 24 weeks of age. Quantitative analysis of angiogenesis- and fibrosis-related gene expression in glomeruli identified by the LMD method in $d b / m$ and $d b / d b$ mice at 24 weeks of age. (a) LRG1, (b) VEGF, (c) VEGFR-2, (d) TGF- $\beta$, (e) Collagen IV, (f) PAI-1, (g) ENG, and (h) T $\beta$ RII. Values are expressed as the mean \pm SEM ( $\mathrm{n}=4-6$ in each group). $* P<0.05, * * P<0.01$ versus $d b / m$ mice, unpaired $t$-test. 
addition, the glomerular expression levels of fibrosis-related genes, such as TGF- $\beta$, Collagen IV, and PAI-1, were significantly increased in $d b / d b$ mice compared with $d b / m$ mice (Figures 5(d)-5(f)). Similarly, glomerular mRNA expression levels of ENG and T $\beta$ RII were significantly increased in $d b / d b$ mice compared with $d b / m$ mice (Figures $5(\mathrm{~g})$ and 5(h)). These results indicated that diabetic nephropathy had developed further after the preceding increase in glomerular LRG1 expression of $d b / d b$ mice.

\section{Discussion}

In the present study, we investigated the intrarenal expression and distribution of a novel proangiogenic factor, LRG1, along with the development of early stage diabetic nephropathy. Our most important finding is that the increase in LRG1 expression of glomerular endothelial cells precedes the increase in VEGF expression, which is another proangiogenic factor involved in the development of diabetic nephropathy. This finding suggests that LRG1 plays a major role in the initial development of diabetic nephropathy by promoting abnormal angiogenesis and glomerular hypertrophy.

In diabetic $d b / d b$ mice at 16 and 24 weeks of age, they exhibited moderate increases in albuminuria levels and glomerular hypertrophy without nodular glomerulosclerosis. These data indicate that our diabetic mice represented the features of early stage diabetic nephropathy, as reported previously [27]. Notably, at 16 weeks of age, glomerular LRG1 expression in diabetic mice was elevated despite glomerular VEGF expression being not increased yet. Considering the results indicating that the glomerular volume and endothelial cells were significantly increased in diabetic mice at this early age, abnormal angiogenesis would already exist in the glomerulus. Thus, there is the possibility that the abnormal angiogenesis observed in the very early stage of diabetic nephropathy in $d b / d b$ mice was attributed to LRG1.

The interaction between LRG1 and VEGF is still unclear, but some studies have shown a possible link between these two factors. In the retina of LRG1 knockout mice, VEGF expression is significantly decreased compared with wildtype mice [15]. In colorectal cancer cells, LRG1 directly induces VEGF expression and promotes tumor angiogenesis [28]. However, LRG1 also exerts proangiogenic effects independently of the VEGF signaling pathway. Double blockade of LRG1 and VEGF suppresses angiogenesis more efficiently than single blockade in the mouse model of choroidal neovascularization [15]. In this study, we demonstrated for the first time that glomerular LRG1 expression was increased earlier than VEGF and VEGFR-2 expression under the conditions of pathological angiogenesis in the early stage of diabetic nephropathy. This finding suggests that glomerular LRG1 is involved in the pathogenesis of abnormal angiogenesis independently of the VEGF signaling pathway, at least in the very early stage of diabetic nephropathy.

Although LRG1 binds directly to ENG and activates the ALK1-Smad1/5/8 pathway for angiogenesis, the TGF- $\beta$ mediated Smad2/3 pathway is subsequently activated as a counterbalance against angiogenesis to induce quiescence in the endothelium $[29,30]$. This vicious pathological cycle of angiogenesis and quiescence finally leads to glomerular fibrosis in diabetic nephropathy [31-34]. In our study, 24-weekold $d b / d b$ mice exhibited upregulation of glomerular fibrosisrelated genes, which was concomitant with glomerular hypertrophy and endothelial cell proliferation. It is assumed that the LRG1-induced abnormal angiogenesis observed in 16week-old $d b / d b$ mice resultantly led to glomerular fibrosis at the later stage.

Our study did not demonstrate whether the increased glomerular LRG1 expression actually caused the abnormal angiogenesis in diabetic $d b / d b$ mice. Therefore, the functional role of LRG1 in abnormal angiogenesis in the early stage of diabetic nephropathy must be investigated further using LRG1 transgenic and knockout mice. Nevertheless, the findings of the present study provide important information regarding the pathogenesis of abnormal angiogenesis in early stage diabetic nephropathy, suggesting that LRG1 is a novel preemptive therapeutic target in diabetic nephropathy.

\section{Conclusions}

Diabetic $d b / d b$ mice exhibited glomerular hypertrophy with abnormal angiogenesis characterized by endothelial cell proliferation at 16 weeks of age, concomitant with an increase in LRG1 expression of glomerular endothelial cells. However, glomerular VEGF expression was not increased at this early stage. The features of early diabetic nephropathy in $d b / d b$ mice had developed further, along with further enhanced glomerular LRG1 expression at 24 weeks of age. Glomerular VEGF and fibrosis-related-gene expression was also significantly increased at this late stage compared with nondiabetic $\mathrm{db} / \mathrm{m}$ mice. These data demonstrate that LRG1 might be pivotal for the initial development of diabetic nephropathy by promoting abnormal angiogenesis.

\section{Data Availability}

The data used to support the findings of this study are included within the article.

\section{Conflicts of Interest}

The authors declare no conflicts of interest.

\section{Authors' Contributions}

Sona haku and Hiromichi Wakui designed and conducted the study. Sona haku, Hiromichi Wakui, and Kengo Azushima wrote the manuscript. Sona haku, Hiromichi Wakui, Kengo Azushima, Kotaro Haruhara, Sho Kinguchi, Kohji Ohki, Kazushi Uneda, Ryu Kobayashi, Miyuki Matsuda, Takahiro Yamaji, Takayuki Yamada, Shintaro Minegishi, Tomoaki Ishigami, Akio Yamashita, and Kenichi Ohashi performed the experiments. Sona haku, Hiromichi Wakui, and Kotaro Haruhara analyzed the data. Akio Yamashita, Kenichi Ohashi, and Kouichi Tamura supervised the study. All authors approved the final manuscript. 


\section{Acknowledgments}

This work was supported by grants from the Yokohama Foundation for Advancement of Medical Science, a Uehara Memorial Foundation grant, Grants-in-Aid for Scientific Research from the Japan Society for the Promotion of Science, grants from SENSHIN Medical Research, the Banyu Life Science Foundation International, and Salt Science Research Foundation (18C4), and a grant-in-aid from the Cardiovascular Research Fund, Tokyo, Japan. This research was also supported by the grant of Strategic Research Project of Yokohama City University, Japan Agency for Medical Research and Development (AMED), and by The Translational Research program; Strategic PRomotion for practical application of INnovative medical Technology (TR-SPRINT) from AMED. The authors thank Emi Maeda for technical assistance.

\section{References}

[1] O. Gheith, N. Othman, N. Nampoory et al., "Diabetic kidney disease: world wide difference of prevalence and risk factors," Journal of Nephropharmacology, vol. 5, no. 1, pp. 49-56, 2016.

[2] S. A. Johnson and R. F. Spurney, "Twenty years after ACEIs and ARBs: emerging treatment strategies for diabetic nephropathy," American Journal of Physiology-Renal Physiology, vol. 309, no. 10, pp. F807-F820, 2015.

[3] M. Guo, S. D. Ricardo, J. A. Deane, M. Shi, L. Cullen-McEwen, and J. F. Bertram, "A stereological study of the renal glomerular vasculature in the $\mathrm{db} / \mathrm{db}$ mouse model of diabetic nephropathy," Journal of Anatomy, vol. 207, no. 6, pp. 813-821, 2005.

[4] K. Ichinose, Y. Maeshima, Y. Yamamoto et al., "2-(8-Hydroxy6-methoxy-1-oxo-1H-2-benzopyran-3-yl) propionic acid, an inhibitor of angiogenesis, ameliorates renal alterations in obese type 2 diabetic mice," Diabetes, vol. 55, no. 5, pp. 1232-1242, 2006.

[5] R. Østerby and G. Nyberg, "New vessel formation in the renal corpuscles in advanced diabetic glomerulopathy," Journal of Diabetic Complications, vol. 1, no. 4, pp. 122-127, 1987.

[6] T. Nakagawa, T. Kosugi, M. Haneda, C. J. Rivard, and D. A. Long, "Abnormal angiogenesis in diabetic nephropathy," Diabetes, vol. 58, no. 7, pp. 1471-1478, 2009.

[7] A. Dei Cas and L. Gnudi, "VEGF and angiopoietins in diabetic glomerulopathy: how far for a new treatment?" Metabolism Clinical and Experimental, vol. 61, no. 12, pp. 1666-1673, 2012.

[8] R. Østerby, A. Hartmann, and H.-J. Bangstad, "Structural changes in renal arterioles in type I diabetic patients," Diabetologia, vol. 45, no. 4, pp. 542-549, 2002.

[9] R. Østerby, "Glomerular structural changes in Type 1 (insulindependent) diabetes mellitus: causes, consequences, and prevention," Diabetologia, vol. 35, no. 9, pp. 803-812, 1992.

[10] D. H. Kang, J. Hughes, M. Mazzali et al., "Impaired angiogenesis in the remnant kidney model: II. Vascular endothelial growth factor administration reduces renal fibrosis and stabilizes renal function," Biomed Research International, vol. 12, no. 7, pp. 14481457, 2001.

[11] D. Kang, Y. Kim, T. F. Andoh et al., "Post-cyclosporinemediated hypertension and nephropathy: amelioration by vascular endothelial growth factor," American Journal of Physiology-Renal Physiology, vol. 280, no. 4, pp. F727-F736, 2001.
[12] T. Ostendorf, U. Kunter, F. Eitner et al., "VEGf ${ }_{165}$ mediates glomerular endothelial repair," The Journal of Clinical Investigation, vol. 104, no. 7, pp. 913-923, 1999.

[13] Katsuyuk Tanabe I, Yohe Maeshima I, Yasufum Sato I, and Jun Wada, "Antiangiogenic therapy for diabetic nephropathy," BioMed Research International, vol. 2017, Article ID 5724069, 12 pages, 2017.

[14] R. A. Kowluru, Q. Zhong, and J. M. Santos, "Matrix metalloproteinases in diabetic retinopathy: potential role of MMP-9," Expert Opinion on Investigational Drugs, vol. 21, no. 6, pp. 797805, 2012.

[15] X. Wang, S. Abraham, J. A. McKenzie et al., "LRG1 promotes angiogenesis by modulating endothelial TGF- $\beta$ signalling," Nature, vol. 499, no. 7458, pp. 306-311, 2013.

[16] J. Fu, C. Wei, W. Zhang et al., "Gene expression profiles of glomerular endothelial cells support their role in the glomerulopathy of diabetic mice," Kidney International, vol. 94, no. 2, pp. 326-345, 2018.

[17] A. Maeda, K. Tamura, H. Wakui et al., "Angiotensin receptorbinding protein ATRAP/Agtrap inhibits metabolic dysfunction with visceral obesity," Journal of the American Heart Association, vol. 2, no. 4, Article ID e000312, 2013.

[18] K. Azushima, K. Ohki, H. Wakui et al., "Adipocyte-Specific Enhancement of Angiotensin II Type 1 Receptor-Associated Protein Ameliorates Diet-Induced Visceral Obesity and Insulin Resistance," Journal of the American Heart Association, vol. 6, no. 3, Article ID e004488, 2017.

[19] K. Uneda, H. Wakui, A. Maeda et al., "Angiotensin II Type 1 Receptor-Associated Protein Regulates Kidney Aging and Lifespan Independent of Angiotensin," Journal of the American Heart Association, vol. 6, no. 8, Article ID e006120, 2017.

[20] M. Ohsawa, K. Tamura, H. Wakui et al., "Deletion of the angiotensin II type 1 receptor-associated protein enhances renal sodium reabsorption and exacerbates angiotensin II-mediated hypertension," Kidney International, vol. 86, no. 3, pp. 570-581, 2014.

[21] H. Wakui, T. Dejima, and K. Tamura, "Activation of angiotensin II type 1 receptor-associated protein exerts an inhibitory effect on vascular hypertrophy and oxidative stress in angiotensin IImediated hypertension," Cardiovascular Research, vol. 100, 3, pp. 511-519, 2013.

[22] K. Haruhara, H. Wakui, K. Azushima et al., "Angiotensin receptor-binding molecule in leukocytes in association with the systemic and leukocyte inflammatory profile," Atherosclerosis, vol. 269, pp. 236-244, 2018.

[23] R. Kobayashi, H. Wakui, K. Azushima et al., "An angiotensin II type 1 receptor binding molecule has a critical role in hypertension in a chronic kidney disease model," Kidney International, vol. 91, no. 5, pp. 1115-1125, 2017.

[24] S. Minegishi, T. Ishigami, T. Kino et al., "An isoform of Nedd4-2 is critically involved in the renal adaptation to high salt intake in mice," Scientific Reports, vol. 6, no. 1, Article ID 27137, 2016.

[25] H. Wakui, K. Tamura, S.-I. Masuda et al., "Enhanced angiotensin receptor-associated protein in renal tubule suppresses angiotensin-dependent hypertension," Hypertension, vol. 61, no. 6, pp. 1203-1210, 2013.

[26] J. R. Nyengaard, A. Flyvbjerg, and R. Rasch, "The impact of renal growth, regression and regrowth in experimental diabetes mellitus on number and size of proximal and distal tubular cells in the rat kidney," Diabetologia, vol. 36, no. 11, pp. 1126-1131, 1993. 
[27] K. Azushima, S. B. Gurley, and T. M. Coffman, "Modelling diabetic nephropathy in mice," Nature Reviews Nephrology, vol. 14, no. 1, pp. 48-56, 2017.

[28] J. Zhang, L. Zhu, J. Fang, Z. Ge, and X. Li, "LRG1 modulates epithelial-mesenchymal transition and angiogenesis in colorectal cancer via HIF-1 $\alpha$ activation," Journal of Experimental \& Clinical Cancer Research, vol. 35, no. 1, 29 pages, 2016.

[29] E. Pardali, M.-J. Goumans, and P. ten Dijke, "Signaling by members of the TGF- $\beta$ family in vascular morphogenesis and disease," Trends in Cell Biology, vol. 20, no. 9, pp. 556-567, 2010.

[30] M.-J. Goumans, G. Valdimarsdottir, S. Itoh, A. Rosendahl, P. Sideras, and P. Ten Dijke, "Balancing the activation state of the endothelium via two distinct TGF- $\beta$ type I receptors," $E M B O$ Journal, vol. 21, no. 7, pp. 1743-1753, 2002.

[31] F. N. Ziyadeh, B. B. Hoffman, D. C. Han et al., "Long-term prevention of renal insufficiency, excess matrix gene expression, and glomerular mesangial matrix expansion by treatment with monoclonal antitransforming growth factor-beta antibody in $\mathrm{db} / \mathrm{db}$ diabetic mice," Proceedings of the National Acadamy of Sciences of the United States of America, vol. 97, no. 14, pp. 7667$7669,2000$.

[32] S. Won Hong, M. Isono, S. Chen, M. C. Iglesias-de la Cruz, D. C. Han, and F. N. Ziyadeh, "Increased Glomerular and Tubular Expression of Transforming Growth Factor- $\beta 1$, Its Type II Receptor, and Activation of the Smad Signaling Pathway in the $\mathrm{db} / \mathrm{db}$ Mouse," The American Journal of Pathology, vol. 158, no. 5, pp. 1653-1663, 2001.

[33] S. Chen, M. C. Iglesias-de la Cruz, B. Jim, S. W. Hong, M. Isono, and F. N. Ziyadeh, "Reversibility of established diabetic glomerulopathy by anti-TGF- $\beta$ antibodies in $\mathrm{db} / \mathrm{db}$ mice," Biochemical and Biophysical Research Communications, vol. 300, no. 1, pp. 16-22, 2003.

[34] Xiaoyun Cheng, Wenke Gao, Yongyan Dang et al., "Both ERK/MAPK and TGF-Beta/Smad Signaling Pathways Play a Role in the Kidney Fibrosis of Diabetic Mice Accelerated by Blood Glucose Fluctuation," Journal of Diabetes Research, vol. 2013, Article ID 463740, 8 pages, 2013. 


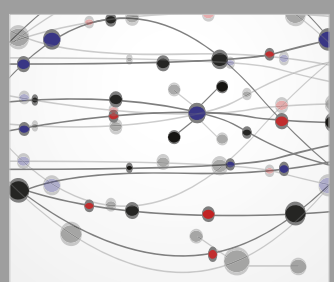

The Scientific World Journal
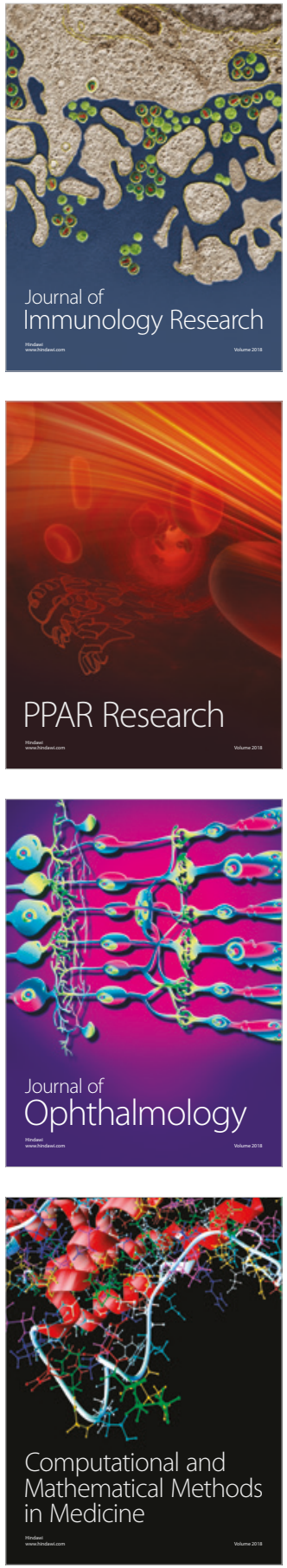

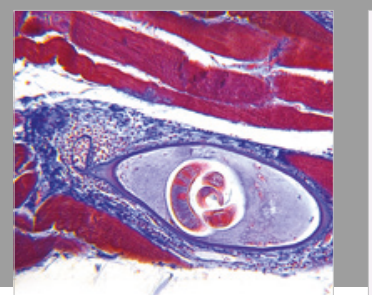

Gastroenterology Research and Practice

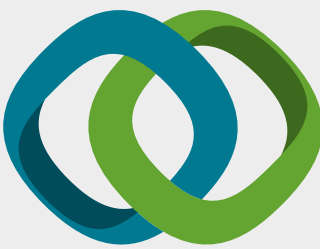

\section{Hindawi}

Submit your manuscripts at

www.hindawi.com
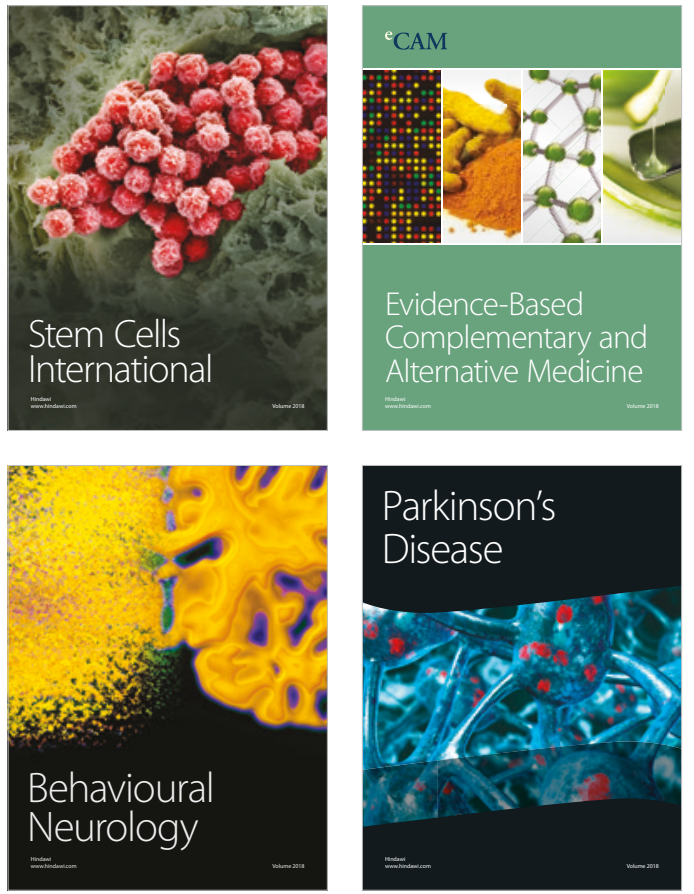

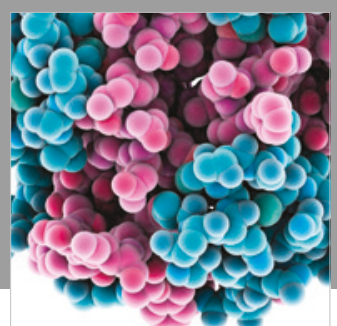

ournal of

Diabetes Research

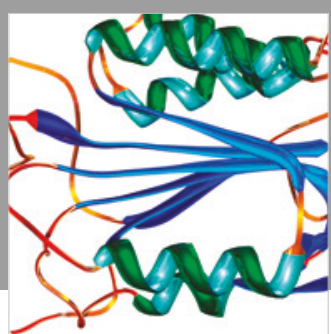

Disease Markers
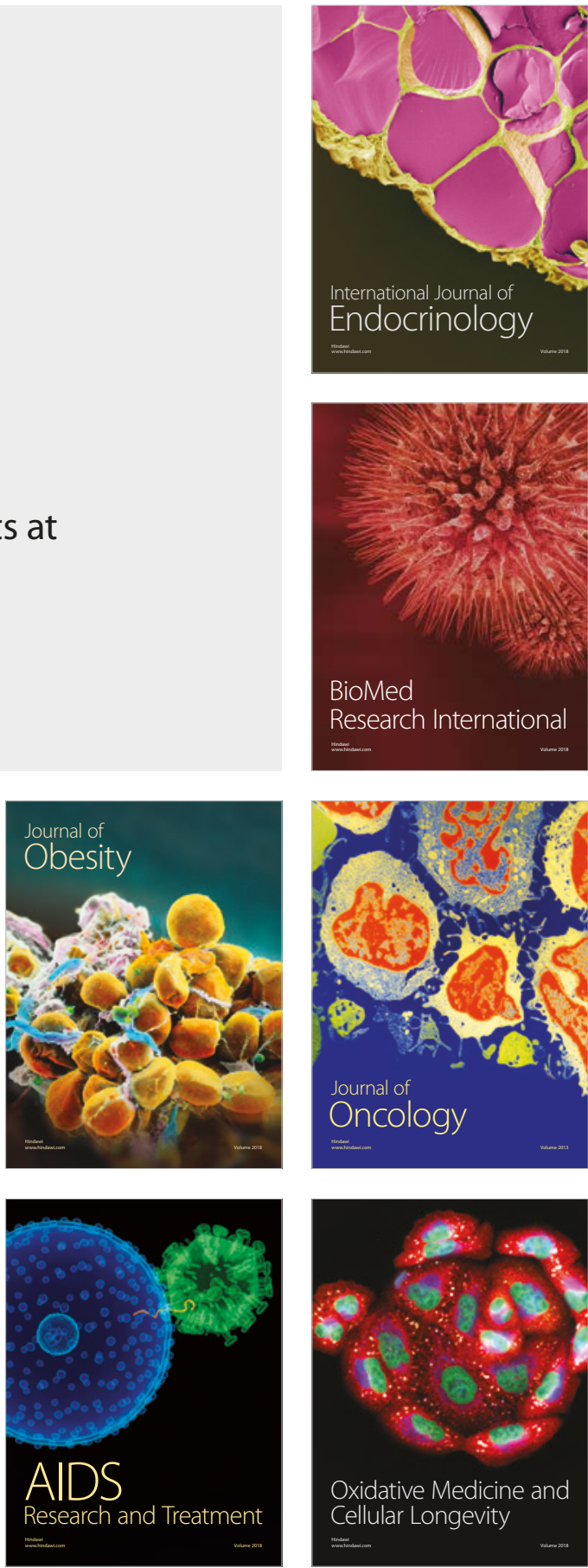